\title{
Design of secure Ad Hoc network using three dimensional discrete wavelet transformation based on performance enhancement
}

\author{
Laith Ali Abdul-Rahaim, Ammar Abdulrasool Muneer \\ Department of Electrical Engineering, University of Babylon, Babil, Iraq
}

Email address:

drlaithanzy@yahoo.com (L. A. Abdul-Rahaim), ammar.abdulrasool@gmail.com (A. A. Muneer)

\section{To cite this article:}

Laith Ali Abdul-Rahaim, Ammar Abdulrasool Muneer. Design of Secure Ad Hoc Network Using Three Dimensional Discrete Wavelet Transformation Based on Performance Enhancement. American Journal of Networks and Communications. Special Issue: Ad Hoc Networks. Vol. 4, No. 3-1, 2015, pp. 35-44. doi: 10.11648/j.ajnc.s.2015040301.17

\begin{abstract}
This work shows new and efficient algorithm of cryptographic purpose based symmetric and conventional techniques that considers the representation of the cipher text by using the three dimensional Discrete Wavelet Transform to find the wavelet decomposition vector containing the approximation and the detail coefficients then build the three dimensional data structure approach. The decryption is done by extracting the encrypted data from the wavelet decomposition vector using the algorithm of inverse Discrete Wavelet Transformation. The encrypted message consists the wavelet decomposition vector. The key is used for authorization purpose to access the network. Results shows great data security and BER over wireless channels based Ad Hoc network.
\end{abstract}

Keywords: 3D-Discrete Wavelet Transform, Cryptography, Signal Processing, Symmetric Cryptography, Wavelet Decomposition

\section{Introduction}

Cryptography is one of the most important tools that provide data and information confidentiality by hiding it. It is usually done through mathematical manipulation of the data with an incomprehensible format for unauthorized users.

In this work, a cryptographic techniques that based on Transformation of Three Dimensional-Discrete Wavelet is presented. Section 2 explains theory of wavelet transformation. Section 3 the process of encryption and decryption of the transmitting of real time information between two clients. Section 4 shows the results of the algorithm using different topologies. Section 5 is an analytic discussion on the technique with the conclusion and future scopes of this work.

\section{The Basics of Wavelet}

Wavelet is mathematical tool that analysis data into different frequency component values, and then check each component with a resolution matched to its scale value. They have benefits over customary Fourier methods in analyzing physical states where the signal has discontinuities and sharp points [1]. Wavelets were advanced individually in the fields of quantum, physics mathematics and electrical engineering. Swaps between these fields during the last thirty years have controlled to many new wavelet uses such as human vision, turbulence, image compression, earthquake prediction, and radar [2, 3].

The necessary idea behind using wavelets is to analyze according to predefined scale value. Definitely, researchers in the wavelet application field sense that, using wavelets, one is implementing a whole new perspective in processing data.

The DWT variables (scale and timing window) are defined as discrete in time and scale, means that the DWT coefficients could have real values (floating-point), but the scale and time values used to guide these coefficients are integers [4, 5].

An information is analyzed by Discrete wavelet transformation into different resolution of one or more levels (called octaves), as presented in Fig. 1, where a 1dimensional signal is analyzed into three octaves. Figure 2 expressed a one-dimensional, one- octave discrete wavelet transform. It contains the decomposing on the left side and the synthesis on the right side. The low-pass filter generates the average signal, while the high-pass filter produces detail 
signal. In multi-resolution analysis, the average signal at one level is sent to another set of filters (Fig. 1), which produces the average and detail signals at the next octave $[6,12]$.

The detail signals are retained, anyway, the higher octave averages could be discarded, because they could be recalculated through the inverse transform process. Every output of channel has only amount of half data input (plus a few coefficients due to the filter process). However, the wavelet illustration is approximately the same size as the original. The discrete wavelet transform can be 1dimensional, 2-D, 3-D, etc. dependent on dimensions of the signal $[7,8]$.

The two dimensional transformation is merely an use of the one dimensional discrete wavelet transformation in the horizontal and vertical dimensions [8]. The illustration in Figure 3 shows the two dimensional transform (separable) for one octave (level). The non-separable two dimensions transform is different from the one shown, since it calculates the transformation based on a two dimensional signal of the input convolved with a matrix, but the outputs are the equal. The separable method could be extended to the three dimensional discrete wavelet transform, as illustrated in Fig.4.

The low-pass filter related to scaling function of the signal, while the high-pass filter related to the wavelet function. The scaling function lets approximation of any given information with a variable value of precision $[9,12]$.

Putting on the below difference equations with the coefficients of scaling function, $h$, gives a calculation of the signal. This is also known as the low-pass output, where $\mathrm{W}$ are the coefficients of scaling function, while $\mathrm{j}$ represents the octave, except in the case of $\mathrm{W}(0, \mathrm{n})$, which is the original signal:

$$
W(j, n)=\sum_{m=0}^{2 n} W(j-1, m) h(2 n-m)
$$

The Convolution with the wavelet function's coefficients, $\mathrm{g}$, produces the detail signal, called high-pass output $\mathrm{W}_{\mathrm{h}}$

$$
W_{h}(j, n)=\sum_{m=0}^{2 n} W(j-1, m) g(2 n-m)
$$

The DWT of a 1-D signal can be computed recursively using a filter pair with the fast pyramid algorithm, by Mallat and Meyer [10], Fig.1. It has a complexity of $\mathrm{O}(\mathrm{N})$, with an input of $\mathrm{N}$ sample. Other transforms normally require $\mathrm{O}(\mathrm{N} 2)$ calculations. Even the Fast Fourier Transform proceeds $\mathrm{O}(\mathrm{N}$ $\log \mathrm{N})$ computations.

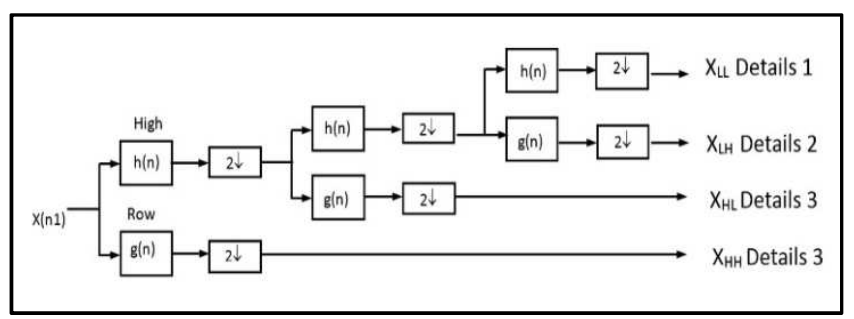

Figure 1. Three Octave of decomposition of a 1-D signal.

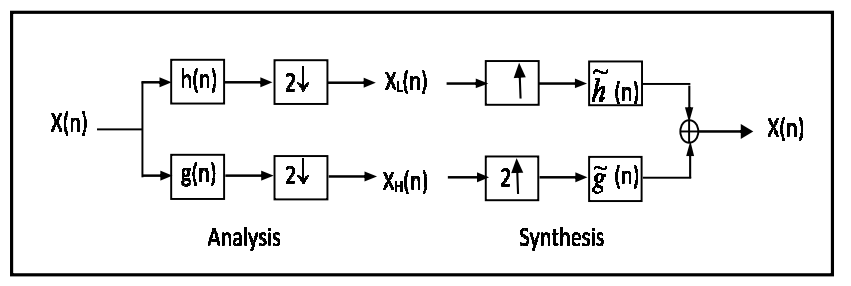

Figure 2. A 1-Dimensional, 1-octave DWT and Inverse DWT.

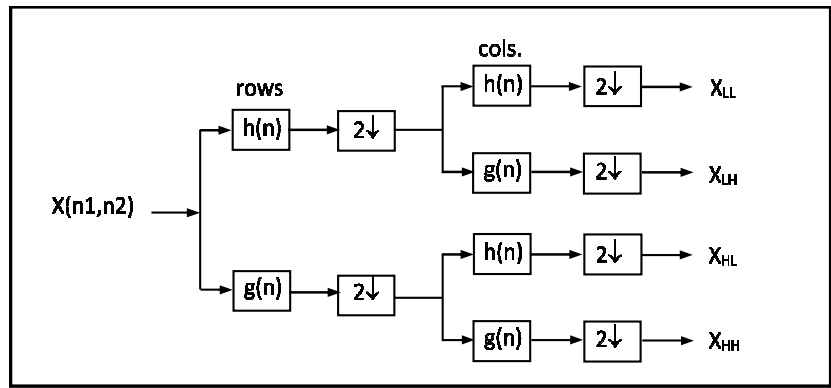

Figure 3. A 2-Dimensional, 1-octave DWT.

The fast pyramid algorithm gets its efficiency by $2^{\mathrm{J}}=$ splitting the output data of each channel, otherwise known as down sampling. Then every octave (levels) uses half the number of data as the previous octave, the maximum number of octaves (levels), J , can be found by setting 2 equal to the input length,i.e. $2^{\mathrm{J}}=\mathrm{N}$, and the discrete wavelet transformation generates approximately $\mathrm{N} / 2 \mathrm{j}$ outputs for each octave j. However, practical using limit the number of octaves (levels) depending on real time processing and other criteria $[10,11]$.

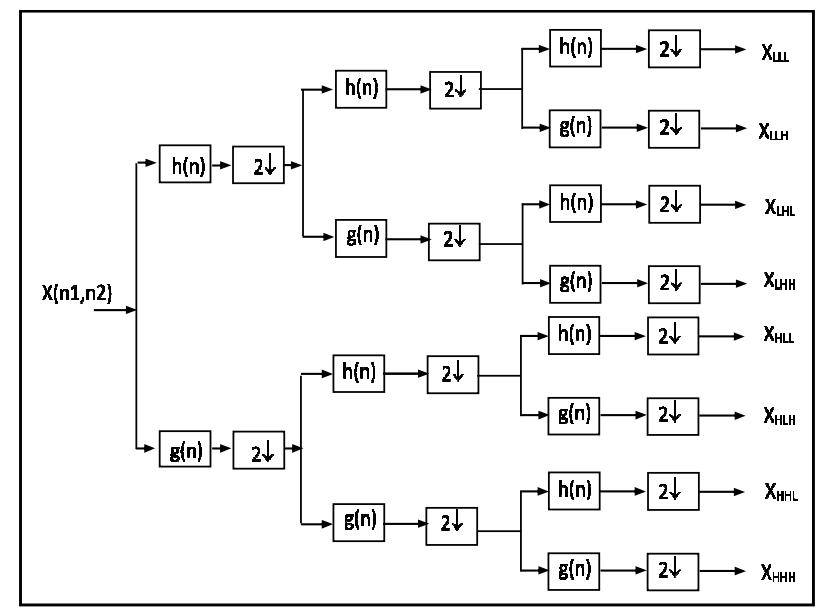

Figure 4. A 3-Dimensional, 1-octave DWT.

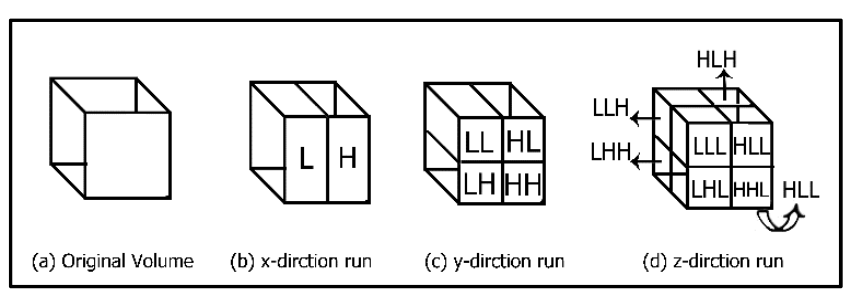

Figure 5. Frequency sub bands produced by single level of wavelet decomposition of a 3-D image. 


\subsection{Prosperities of Wavelets}

Not each waveform provides a wavelet function. Nevertheless, the function should have few characteristics to be a wavelet. Two of the best essential possessions of wavelets are the admissibility and the regularity conditions. It can be shown that square integrable functions $\Psi$ (t) sustaining the admissibility condition which could be used to first analyses and then reconstruct a signal without loss of information content $[12,13]$.

$$
\int \frac{|\psi(\omega)|^{2}}{|\omega|} d \omega \prec+\infty
$$

The above inequality $\Psi(\mathrm{w})$ views the Fourier transform of $\Psi(\mathrm{t})$. The admissibility condition means that the square of Fourier transform of $\Psi(\mathrm{t})$ vanishes at the zero frequency, i.e.

$$
\left.|\psi(\omega)|^{2}\right|_{\omega=0}=0
$$

This means that wavelets must have a band-pass like spectrum. This is a very important observation, which is used to construct efficient wavelet transforms [14]. Furthermore, at the zero frequency also means that the average value of the wavelet in the time domain should be equal to zero and therefore it must be oscillatory (oscillating wave). In other words, $\Psi(\mathrm{t})$ must be a wave in Continuous time domain. Similarly, $\Psi$ (n) will be a wave in discrete time domain. Mathematically,

$$
\int \psi(t) d t=0, \text { and }, \sum \psi(n)=0
$$

In addition, the regularity of wavelet corresponds to the number of vanishing moments [15]. Therefore, if a wavelet has $\mathrm{N}$ vanishing moments, then the approximation order of the wavelet transform is also $\mathrm{N}$ as compared with $\mathrm{N}$ vanishing moment. A small value is often good instead of exactly zero of vanishing moment. The suggestions from experimental research show that the number of vanishing moments required are application dependent. The first moment to be vanished is corresponding to admissibility condition $[16,17,18]$

\section{Encryption and Decryption for Wireless}

The planned DSP based security of communication system and Transmitter and Receiver using UDP protocol, the system is shown as in Figure (6).

At the receiving end, all the steps of proposed algorithm applied but in reversely order to retrieve the original data. However frames are divided into 4096 samples to offer(16! x 16 !) possible permutation for each page. This changeability of the scrambled signal is increased to be (16! x16! x16!) when interring the total frame permutation (the third dimension permutation) is also performed. Not all permutations gives good security quality so the effective permutation used [19]. Fortunately, results offer high security level to the system. The block diagrams of the proposed scrambler and descrambler based on this new scheme are shown in Fig.(7).

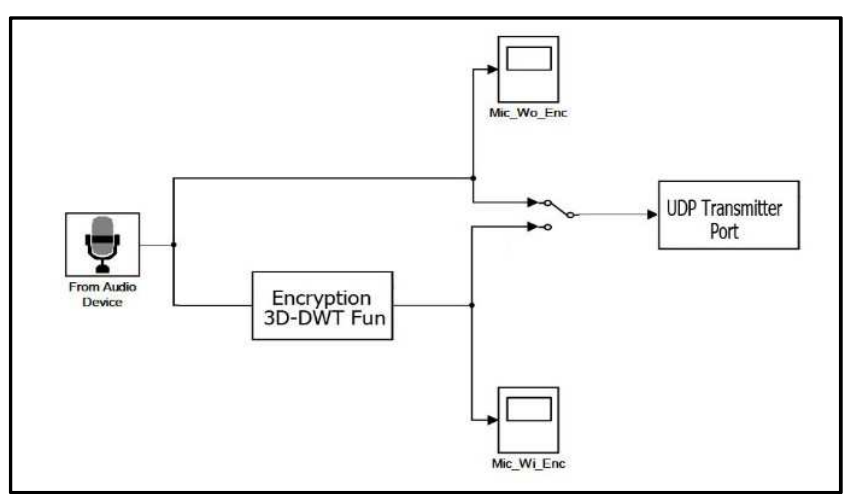

Figure 6-a. Purposed encryption System based Wireless Link (802.11n) over UDP port.

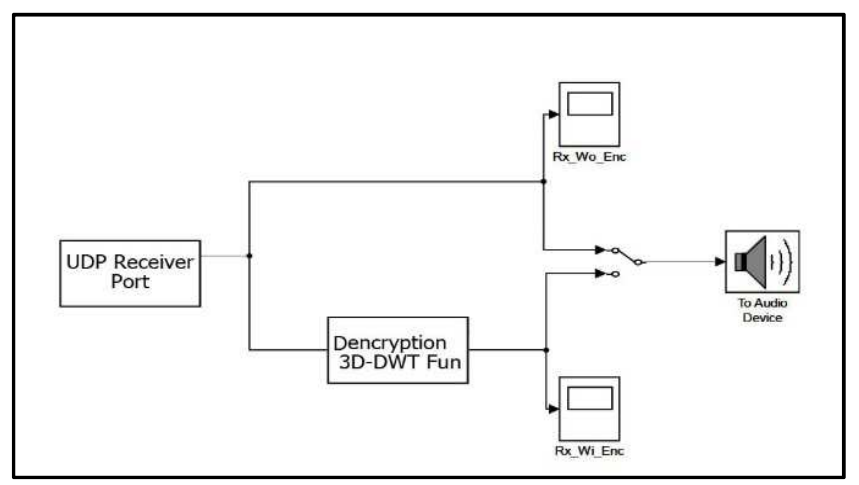

Figure 6-b. Purposed decryption System based Wireless Link (802.11n) over UDP port.

Although using the subject scheme, in order to have insight of time and frequency domain analysis, the recorded wave files of the various speech segments are implemented and evaluated using Simulink MATLAB $\mathbb{R}(\mathrm{R} 2013 \mathrm{a})$. To reinforce the quality of the obtained results, the experiments are conducted not only in English but in Arabic languages as well.

Pauses between talk burst cannot be sensed and there is no residual intelligibility.

In term of security system, the concept of the residual of intelligibility while the quality of the recovered data are subjective quantity, thus the scramble and descramble process techniques are estimated on the results of expert listeners during the test $[20,21,22]$.

Table 1. Purposed System Specifications.

\begin{tabular}{ll}
\hline Parameter & Range \\
\hline Input speech & $300 \mathrm{~Hz}$ to $3400 \mathrm{~Hz}$ \\
Sampling frequency & $44100 \mathrm{~Hz}$ \\
Type of transformation & $3 \mathrm{D}-\mathrm{Discrete}$ Wavelet Transformation \\
Frame length & $4096 \mathrm{sample}$ \\
Frame duration & $92.879 \mathrm{~m} \mathrm{sec}$ \\
Total Changeable coefficients & $16 \times 16 \times 16=4096$ \\
\hline
\end{tabular}

The tests were made tighter by adopting the following steps:

(1) Separating digits that are pronounced via male and 
female as well. (2) Examinations are applied for digits also sentences are included too. (3) Those segments also are proved by male and female too. (4) However, to have more practical results, exchanged segments are carried out via two languages for male and female individually. The results are calculated in terms of correctly identified words Q, which is equal to Q:

$$
Q=\frac{(R-W)}{T} \times 100 \%
$$

Where $\mathrm{R}$, right words, $\mathrm{W}$, wrong words and $\mathrm{T}$ is total words [23].

\begin{tabular}{|c|}
\hline $\begin{array}{c}\text { Capturing and } \\
\text { Sampled Data }\end{array}$ \\
\hline Framing \\
\hline $\begin{array}{c}\text { 1-D to 3-D data } \\
\text { conversion }\end{array}$ \\
\hline 3-D DWT Process \\
\hline Permutation \\
\hline 3-D data to frame \\
conversion
\end{tabular}

\begin{tabular}{|c|}
\hline $\begin{array}{c}\text { Data Received } \\
\text { from UDP port }\end{array}$ \\
\hline $\begin{array}{c}\text { Frame to } 3 \text {-D data } \\
\text { Conversion }\end{array}$ \\
\hline Permutation \\
\hline 3-D IDWT Process \\
\hline 3-D to 1-D data \\
conversion \\
\hline \hline Framing \\
\hline \hline To the Sink Device \\
\hline
\end{tabular}

Figure 7. Encryption algorithm at Transmitter (Red) and Receiver (Blue).

\section{Real Time Wireless Communication}

The below graphs shows the results of real time communication based on Ad-hoc network and star topology using time and spectrum expressions.

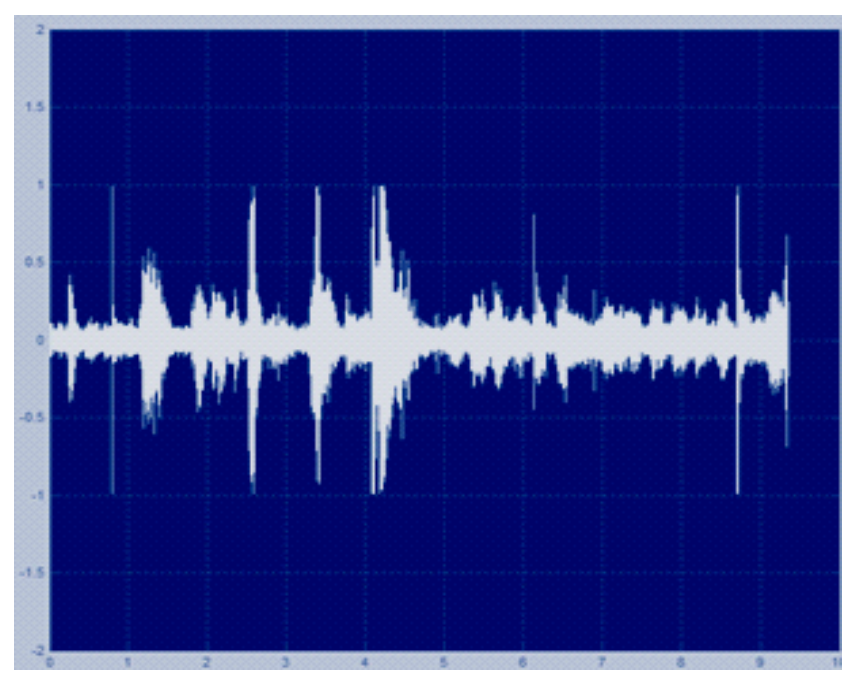

Figure 8. Transmitted data over Ad Hoc network without encryption.

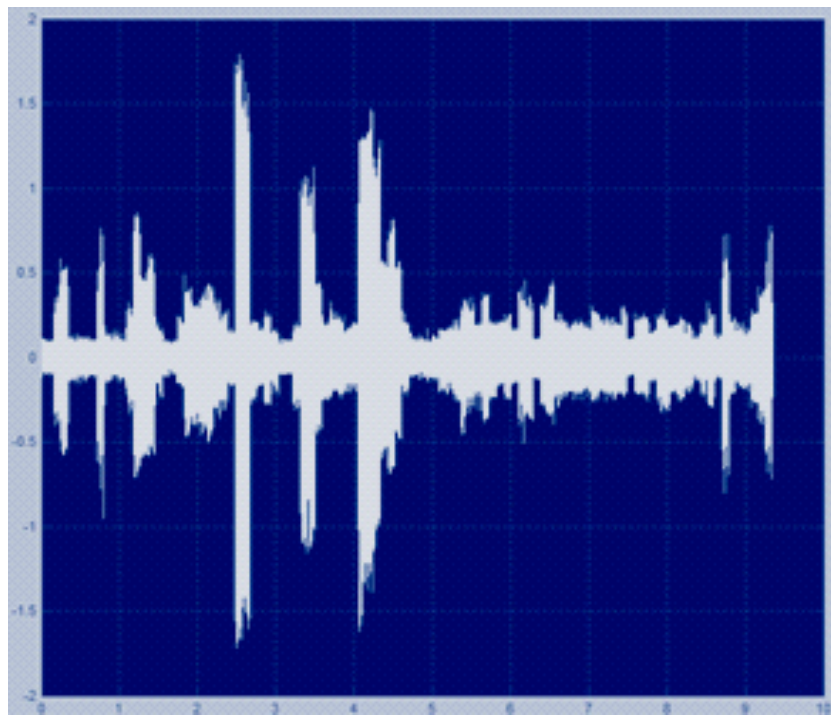

Figure 9. Transmitted data over Ad Hoc network with encryption.

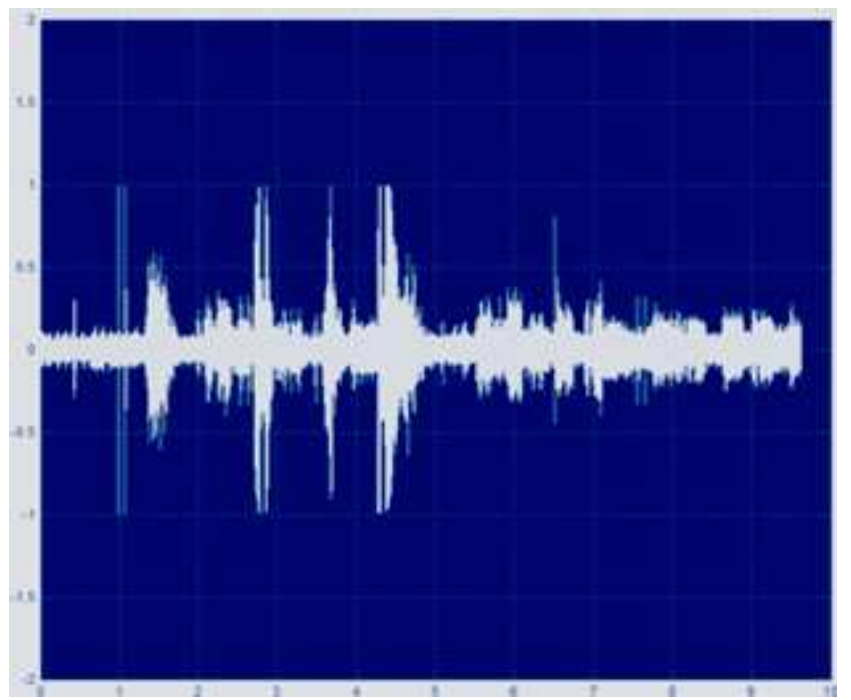

Figure 10. Received data over Ad Hoc network without decryption.

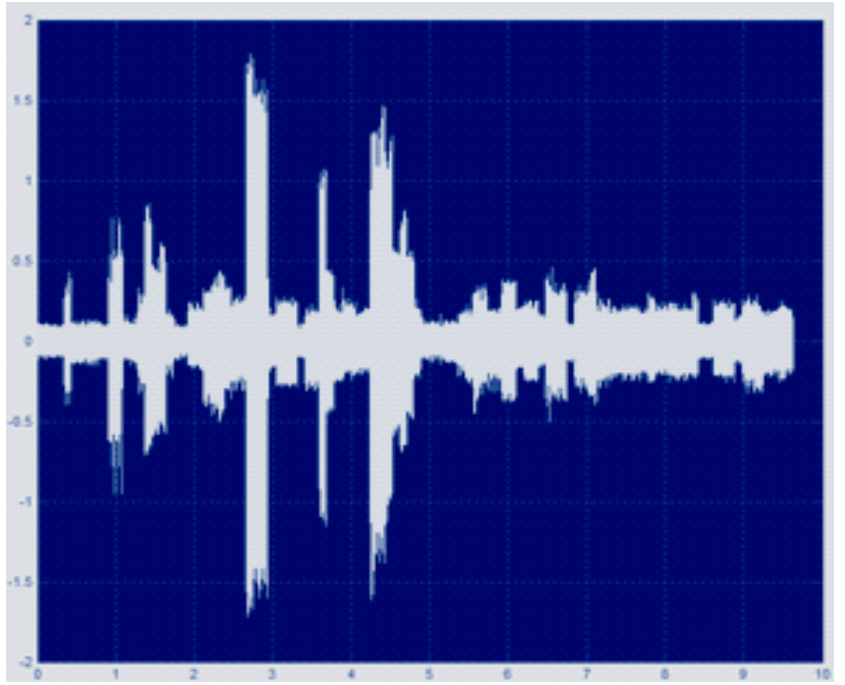

Figure 11. Received data over Ad Hoc network with decryption. 


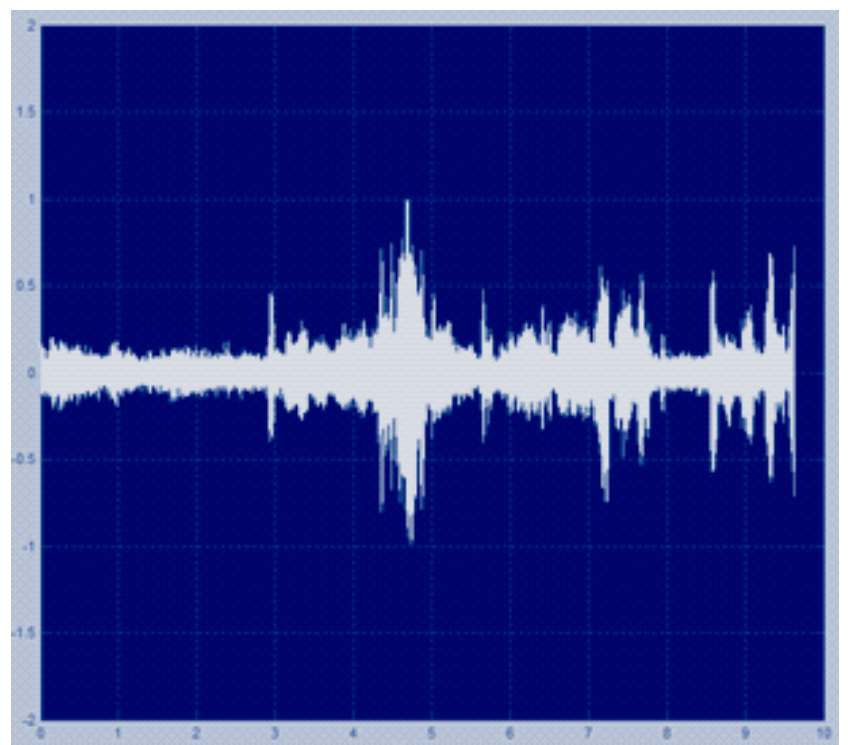

Figure 12. Transmitted data over Star network without encryption.

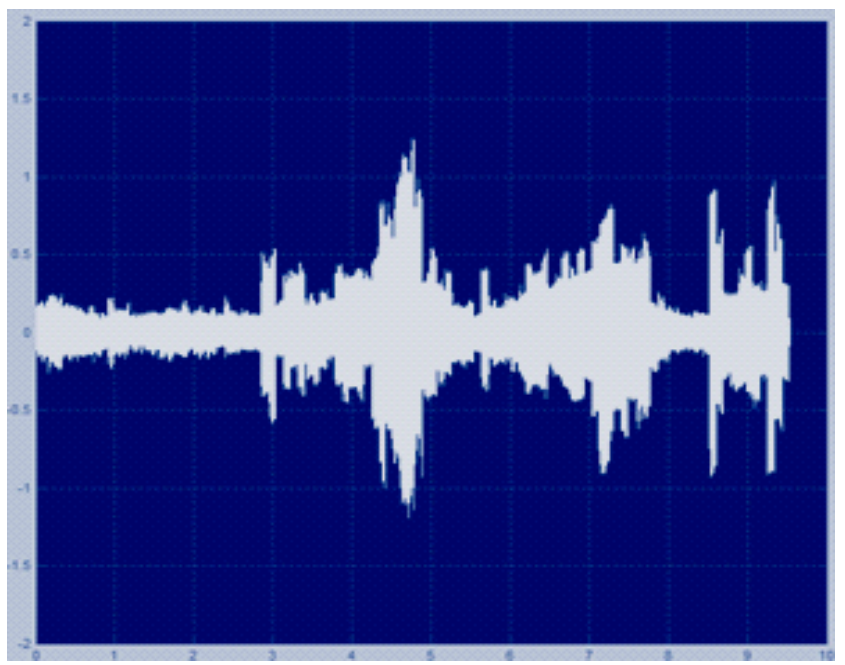

Figure 13. Transmitted data over Star network with encryption.

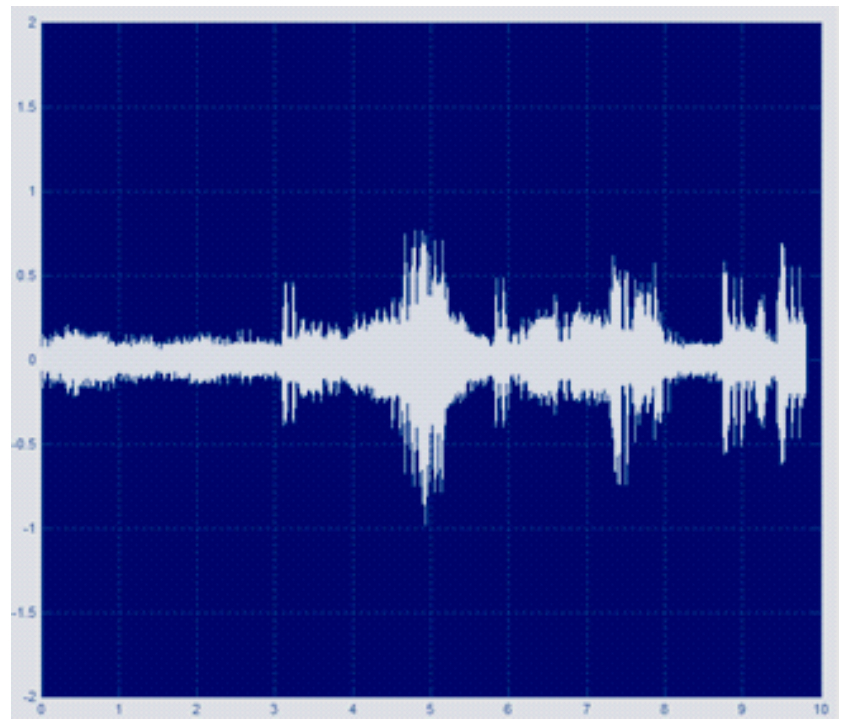

Figure 14. Transmitted data over Star network with encryption.

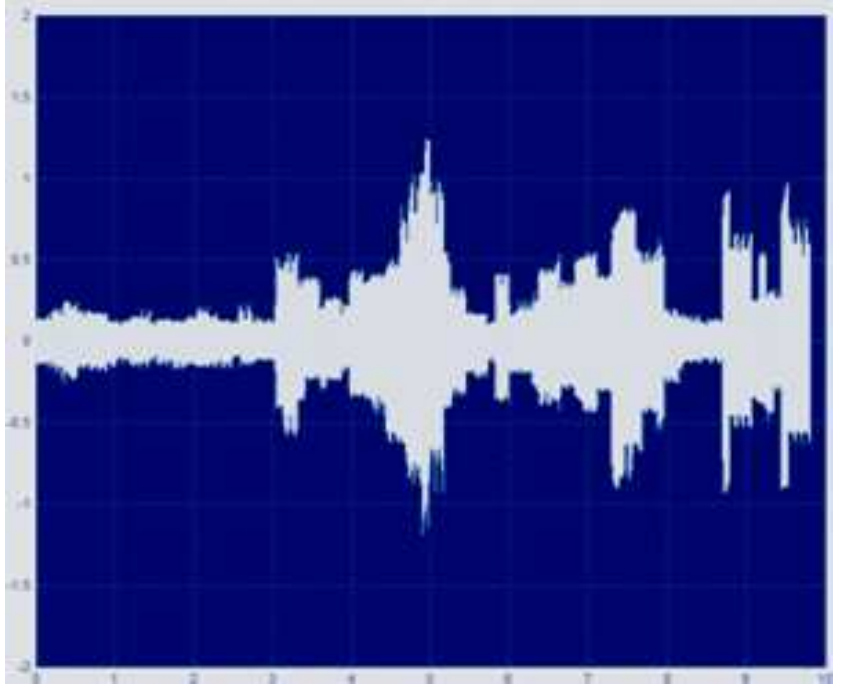

Figure 15. Received data over Star network without decryption.

\section{The Performance Enhancement in Simulation Results}

In this section, the contents show the simulation results of OFDM with proposed encryption based 3D-DWT method.

However, for time-domain it is clearly represented as discrete-time signals. In frequency domain the division of energy is not as original as before the encryption process application. The spectrum is reversed altogether which inverts the distribution of energy level with respect to function of frequency. However, the data is mixed in frequency domain which is similar to convolution in time domain. While transmitted signal is represented in timedomain this leads that any unauthorized access that tries to de-ciphering the data without knowledge about the used scheme, would have to convolve in time-domain which, without doubt would be time consuming process based realtime systems. Furthermore, non- knowledge the permutation order of the system that's why he would have to apply on each frame could be recognized to take infinite time [20,23] as shown in fig.(16).

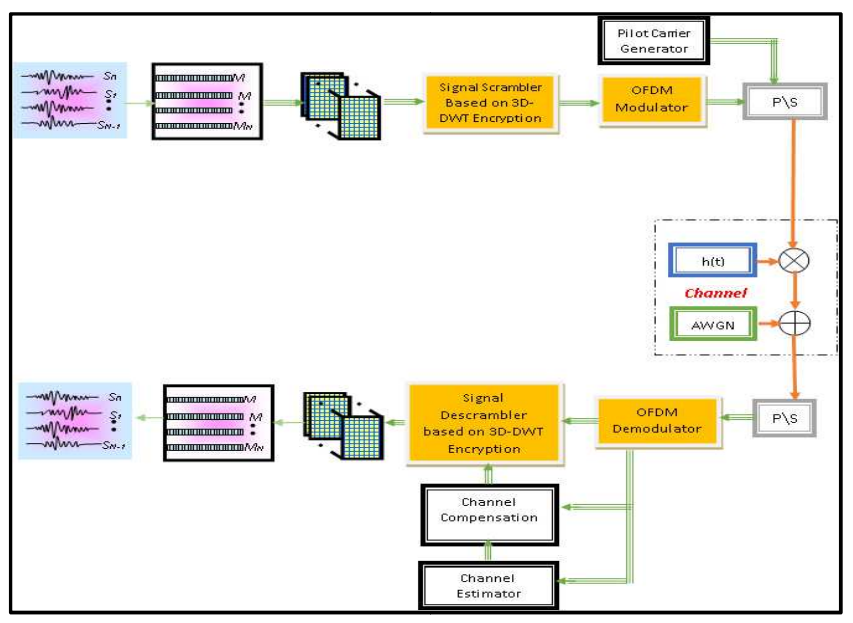

Figure 16. Block diagram of real time encryption system.

These parameters are shown in table (2) 
Table 2. Simulation Parameters.

\begin{tabular}{ll}
\hline $\mathbf{2 5} \mathbf{M H z}$ & Bandwidth \\
\hline AWGN & \\
Flat fading+AWGN & Channel model \\
Frequency selective fading+AWGN & \\
$0.1 \mu \mathrm{sec}$ & Delay spreading $\left(T_{d}\right)$ \\
64 & FFT Points \\
26 & Symbol Number \\
\hline
\end{tabular}

After an extensive tests the results showed over a long period to create those tests clear for listeners, the speech files of that contain wave signals are played and listened by listeners. By following mechanism steps, thirty listeners who are all listened to 50 encrypted wave segments. Segment consists of the digits 0 to 9 is spoken in cluster of four digits. Additional, tests are not restricted to spoken digits only but also to sentence segments. So as to make test stringent and result has oriented feature, the test was hard and consumes time, and the tests are implemented in English as well in Arabic language. However, duplication of spoken digits of the same position is avoided. The tests were inflexible by using:

i. Separating the digits that were spoken by male and female as well.

ii. Test is done for not limited to digits only but also for sentences. The test segments also recorded.

iii. The recorded segments are tested via two languages by male and female.

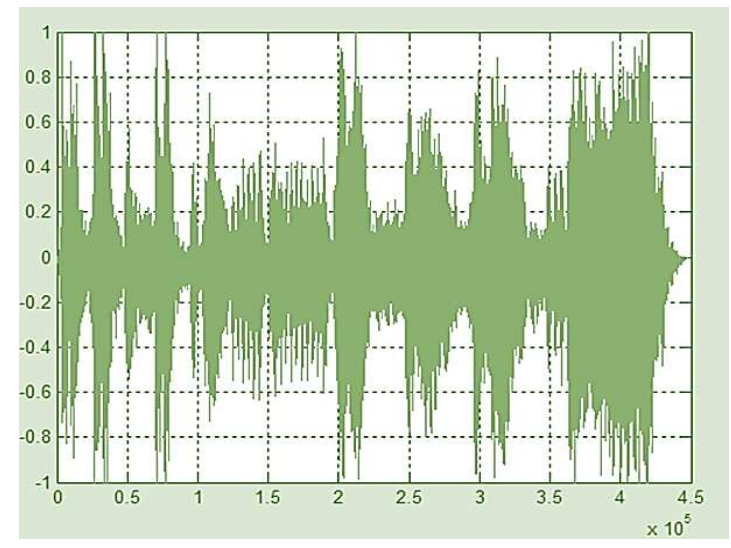

Figure 17. Time Domain of Original Audio File to be encrypted.

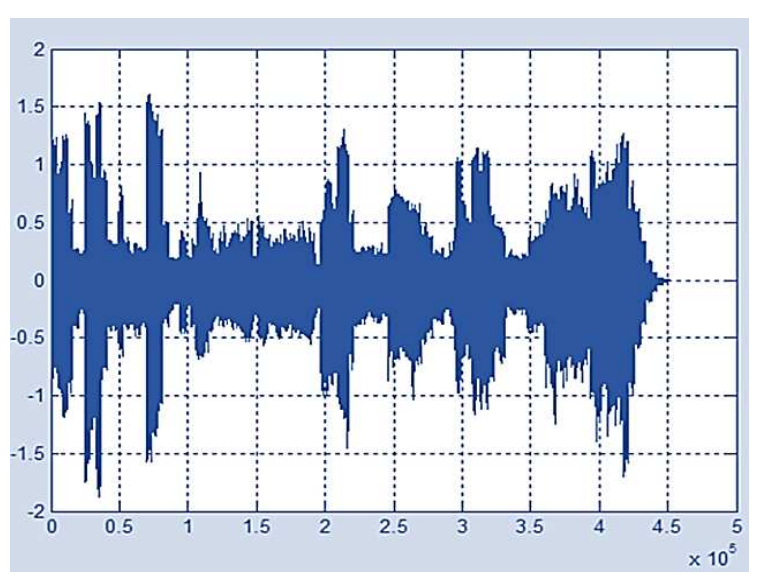

Figure 18. Time Domain of Original Audio File to be encrypted.
The recorded data file, contains spoken digits "Zero, One, Two" vocal by a male is showed in. Figs. (17) - (18) which represent time-domain representation of original ciphered data and deciphered files respectively. On the rest, Figs (20) - (22) reveal distribution of power as a function of frequency of original encrypted and retrieved speech, respectively.

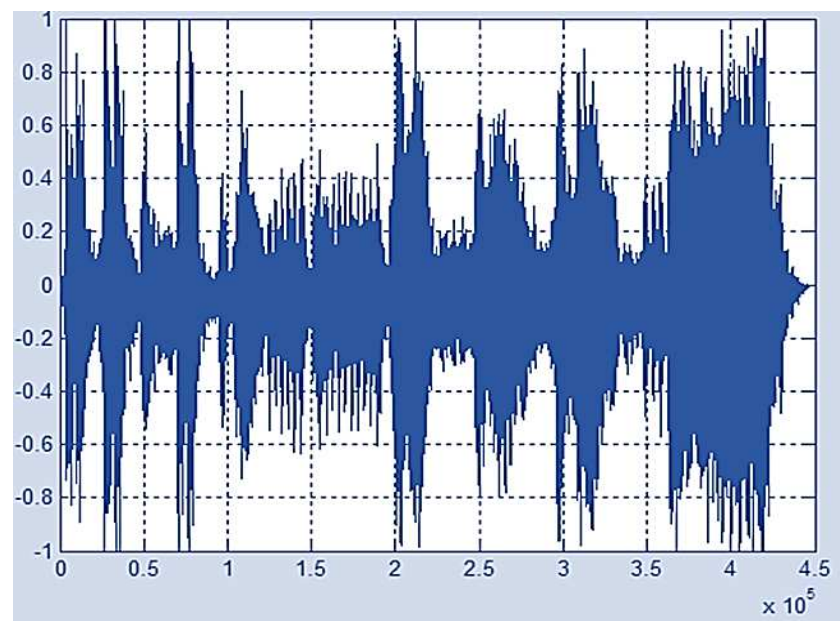

Figure 19. Time Domain of Recovered Audio wave.

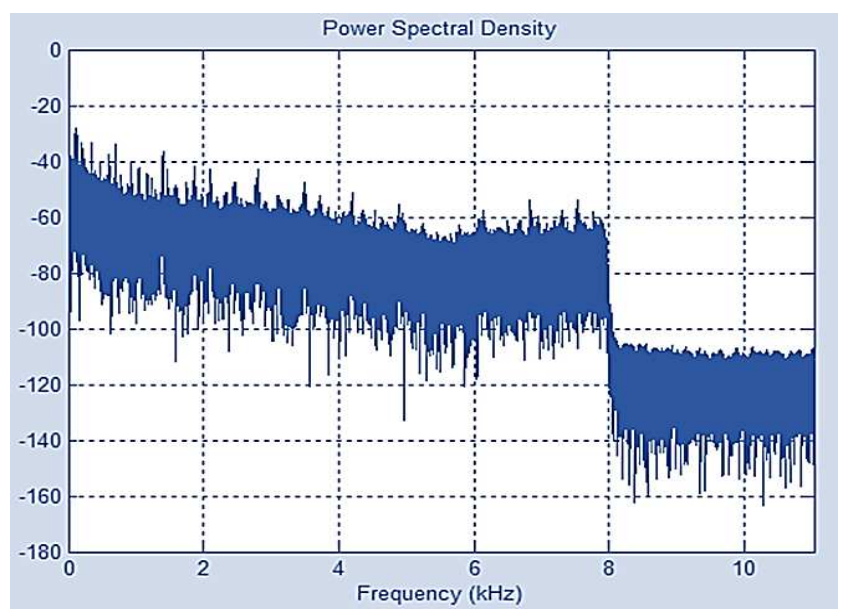

Figure 20. Original Power Spectral Density of Audio wave.

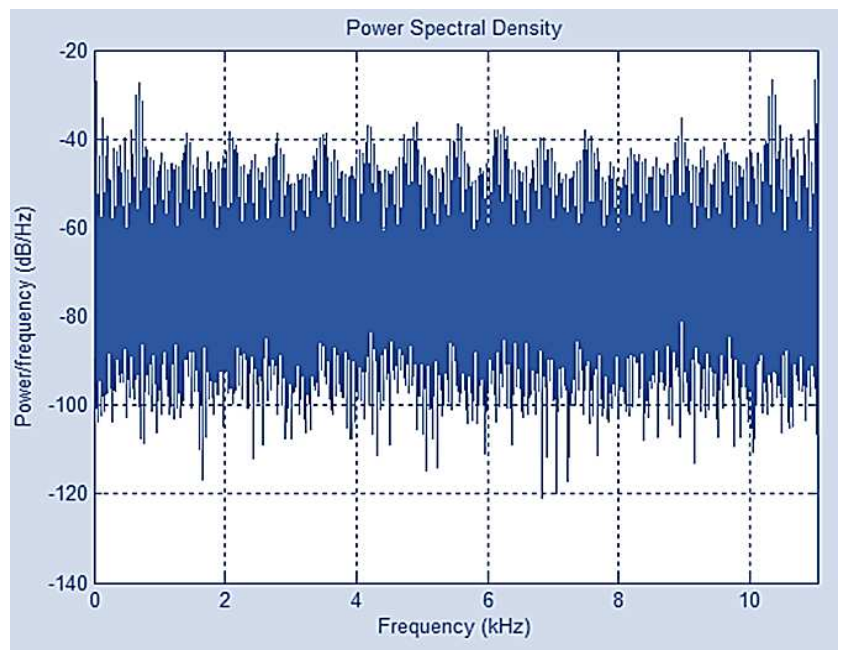

Figure 21. Encrypted Power Spectral Density of Audio wave. 


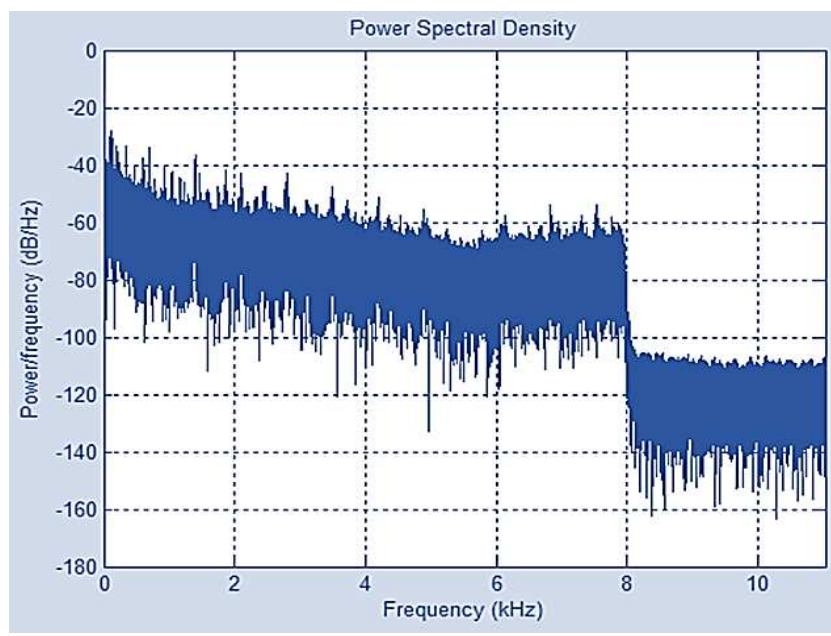

Figure 22. Recovered Power Spectral Density of Audio wave.

\section{A. The Encryption-OFDM In AWGN Channel}

The MATLAB V8.1 is used to simulate the Encryption OFDM transceiver proposed system as shown in Fig.(16). Most MATLAB functions are written to simulate the encryption system as shown in Fig.(16). The functions include frame resizing, Encryption-description, the using of pilot carriers, etc. the output of the simulated proposed system is estimated and represented in Fig.(23), and gives the performance of BER for the Encryption-OFDM using discrete wavelet transformation and OFDM system in AWGN channel. It is represented clearly that the Encryption-OFDM system using 3D-DWT Encryption gives much better results than OFDM transceiver and the Encryption of 2D-DWT OFDM.

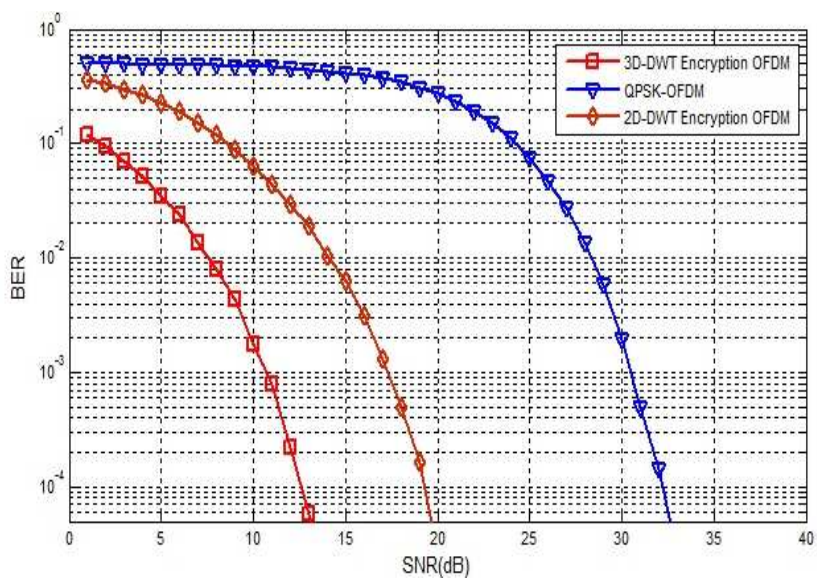

Figure 23. BER performance of Encryption-OFDM using 3D-DWT Encryption in AWGN channel model.

B. The Flat Fading Encryption Channel based 3D-DWT

MATLAB V8.1 simulated the results as in Fig. (16) is used here to mimic the results in flat fading channel additional to AWGN excluding a flat fading channel is added to the channel model. For AWGN and flat fading types of channel, the signal is influenced by the fading effect add to AWGN. However, all the frequency assembled of the signal will be influenced with an attenuation and linear distortion for assumed channel and this leads to a Rayleigh's distribution.

The assumption of $10 \mathrm{~Hz}$ is used for Doppler frequency which leads to BER of 10-4 and the SNR required for Ciphering-OFDM using 3D-DWT is about $17 \mathrm{~dB}$ could be seen from Fig(24), while 2D-DWT OFDM scrambling of transceiver is about $23 \mathrm{~dB}$ and the SNR in OFDM transceiver is about $36 \mathrm{~dB}$.

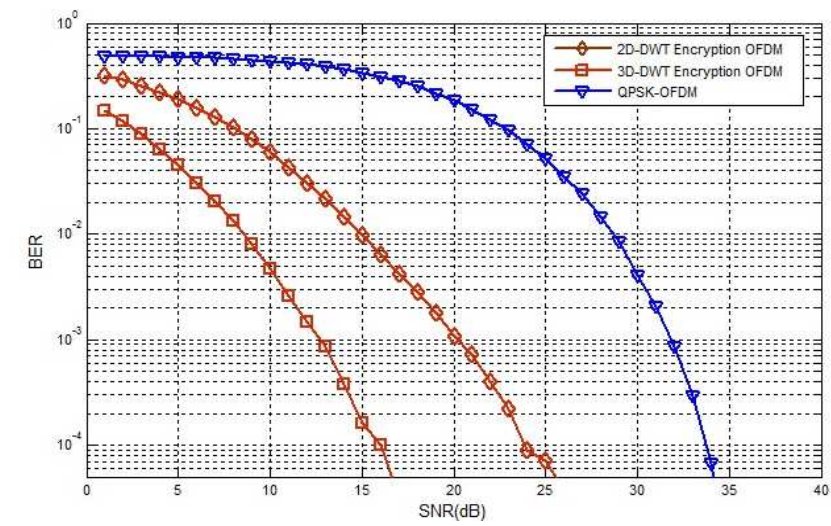

Figure 24. performance of 3D-DWT Encryption for Flat Fading Channel with Doppler Shift $=10 \mathrm{~Hz}$.

The same thing are shown in from fig. (25) and fig. (26), therefore from fig. (24) fig. (25) and fig.(26) a gain of $19 \mathrm{~dB}$ and $6 \mathrm{~dB}$ for the Encryption-OFDM using 3D-DWT Encryption against OFDM 2D-DWT Encryption transceivers are obtained respectively.

Therefore the Encryption-OFDM using 3D-DWT Encryption outdone dramatically for this model channel.

C. The Frequency Selective Fading Channel

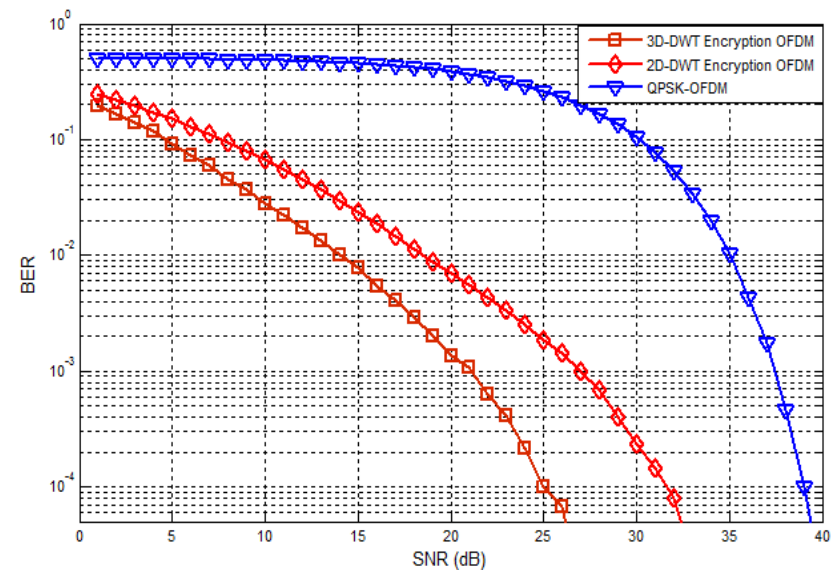

Figure 25. performance of 3D-DWT Encryption for Flat Fading Channel with Doppler Shift $=100 \mathrm{~Hz}$. 


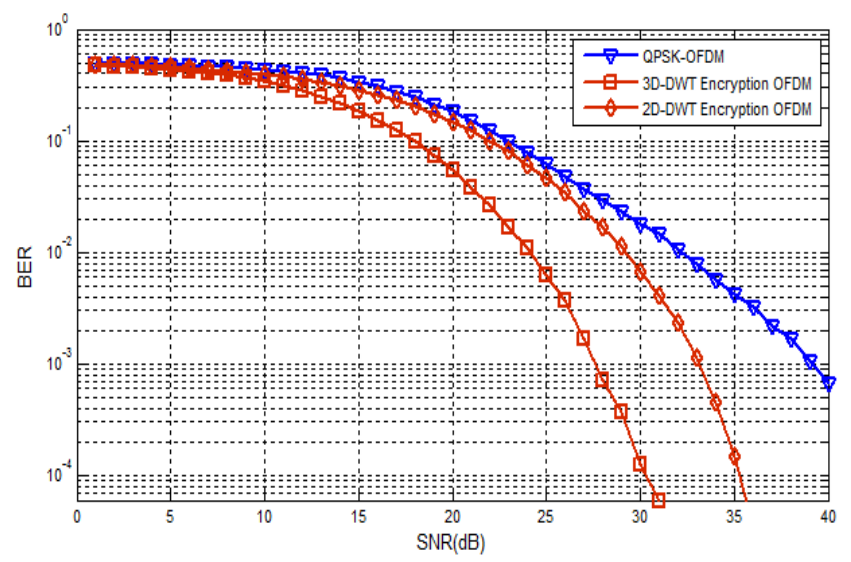

Figure 26. performance of 3D-DWT Encryption for Flat Fading Channel with Doppler Shift $=500 \mathrm{~Hz}$

\section{Encryption based 3D-DWT}

According to BER performances of Encryption-OFDM using 3D-DWT are mimicked for AWGN with multi-path frequency selective Rayleigh distributed channels. Assuming two ray channel with gain of $-8 \mathrm{~dB}$ for the second path, the second path would have maximum delay of $\tau \max =0.1 \mu \mathrm{sec}$ for range of values of signal to the noise ratio. Fig. (27) represents mimic results of fDmax $=10 \mathrm{Hzas}$ maximum Doppler shift. It could be seen clearly from Fig.(27) the $\mathrm{BER}=10 \mathrm{e}-4$ wouldrequire SNR for Encryption-OFDM using 3D-DWT about 19dB, however Encryption-OFDM utilizing 2D-DWT and OFDM transceivers, the SNR areabout $31 \mathrm{~dB}$ and $37 \mathrm{~dB}$ respectively.Therefore from figs.(26) the gain of $18 \mathrm{~dB}$ of the Encryption-OFDM using 3D-DWT against OFDM transceiver which obtained. In Figs(27-29) the same thing can noted that Encryption-OFDM using 3D-DWT Encryption system outperforms significantly for this channel model. In this sections the results are briefed in table (3), also those results are computed later by testing the system via transferring approximately $1 \mathrm{M}$ symbols. Table (3) presents SNR values corresponding to BER.

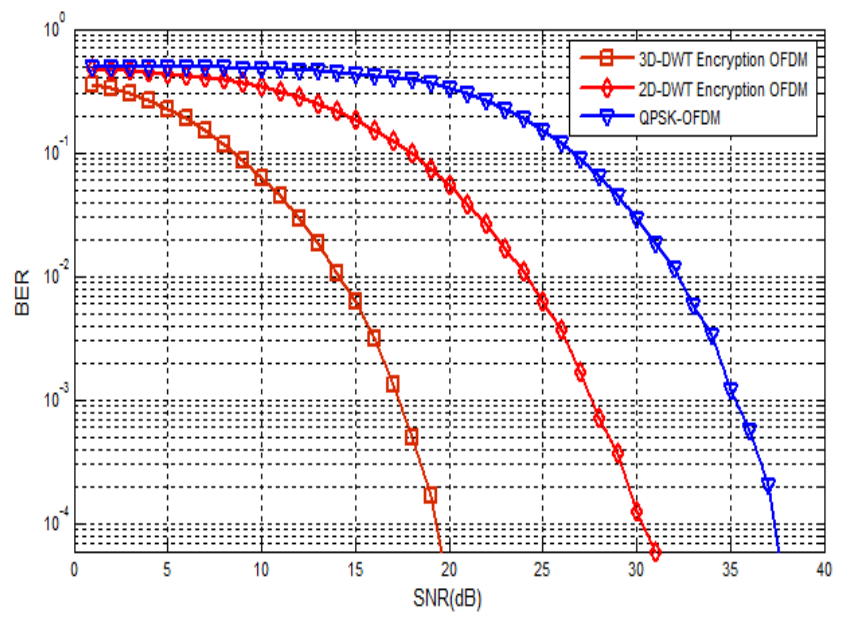

Figure 27. Performance of 3D-DWT Encryption- for Selective Fading Channel with Max. Doppler Shift $=10 \mathrm{~Hz}$.

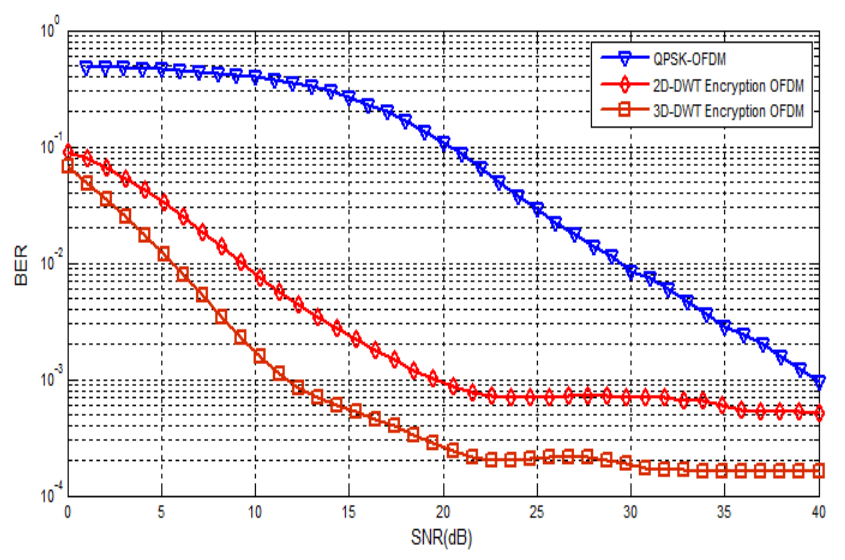

Figure 28. Performance of 3D-DWT Encryption-for Selective Fading Channel with Max. Doppler Shift $=100 \mathrm{~Hz}$.

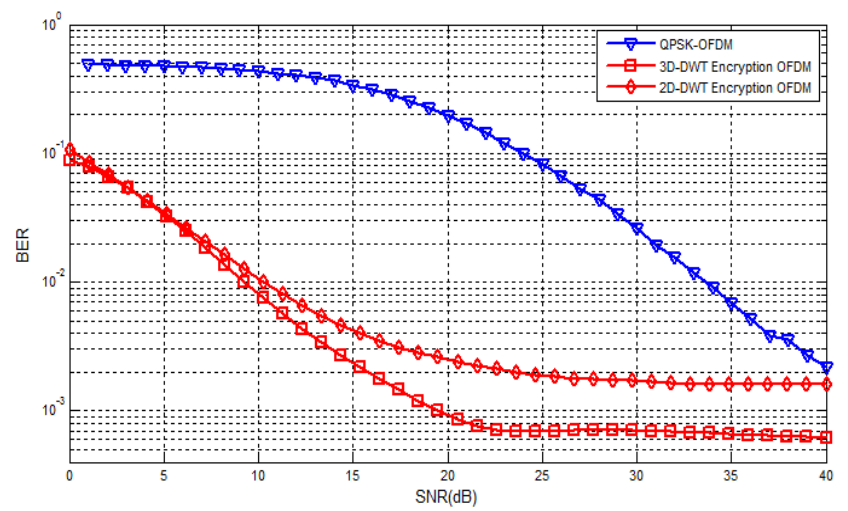

Fig. 29. Performance of 3D-DWT Encryption-for Selective Fading Channel with Max. Doppler Shift $=500 \mathrm{~Hz}$.

Since the essential goal of communication security is the hiding of the fact that a secret message is transmitted, then it is very important to make the recovered process at receiver. we present a list of interpretations.

1. This work is novel in wireless security based DSP techniques, where most algorithms that were used based on inserting dummy packets or based permutation of Fourier Transform, while in this work, Three Dimensional Transformation based Discrete Wavelet Transformation is adapted and has decrease BER over OFDM modulation in different modulated channels as descripted above.

2. It obvious that the proposed system that based on 3DDWT with permutations is secure against brute force attack, when the required process to retrieve data is governed by permutation preprocess it gives system robust against those kind of attacks.

3. The key length based on the length of the message which is close to the best sec urity algorithm (One Time Pad) according to what Shannon showed. The length of the key $=16 \times 16 \times 16=4096=212$.

4. The BER shows great interesting results, the system seems robust and has the immunity for wide range of SNR and this gives two advantages: security and noise immunity.

5. The delay time in Ad hoc network is less $50 \%$ than Star 
network, this results with two nodes. For more than two nodes, this results is reversed, the Star network topology would be faster than Ad hoc, the ration depends on the distance, number of hops between the two nodes, indoor or outdoor, finally, interference existence. The diversity in test shows great results in both topologies with delay distinction in real time environment.

6. The proposed system fulfills most of the Kerchoff's principles which state that the security of the system has to be based on the assumption that the enemy has full knowledge of the design and implementation details of the security system, i.e. the algorithm could be published in public, only the length of key, permutation algorithm and cipher mode type are kept secret, the resultant message is in format which is suitable for transmission, the system is practicallyunbreakable, system implementation is easy and it requires a short time.

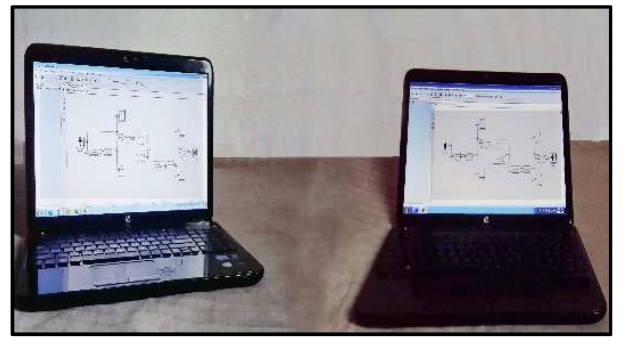

Figure 30. The connection of Two Stations in Ad Hoc topology.

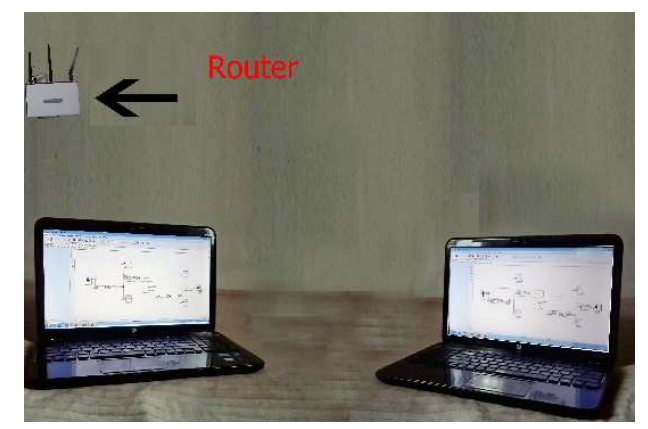

Figure 31. The connection of Two Stations in Star topology.

Table 3. The results for all systems.

\begin{tabular}{|c|c|c|c|c|c|c|c|}
\hline \multirow{3}{*}{ System name } & \multirow{3}{*}{$A W G N$} & \multicolumn{3}{|c|}{ Flat Fading } & \multicolumn{3}{|c|}{ Selective Fading } \\
\hline & & \multicolumn{3}{|c|}{ Max. Doppler Shift } & \multicolumn{3}{|c|}{ Max. Doppler Shift } \\
\hline & & $10 \mathrm{~Hz}$ & $100 \mathrm{~Hz}$ & $500 \mathrm{~Hz}$ & $10 \mathrm{~Hz}$ & $100 \mathrm{~Hz}$ & $500 \mathrm{~Hz}$ \\
\hline OFDM- transceiver & 32 & 33 & 39 & non & 37 & Non & non \\
\hline 2D-DWT ENCRYPTION- transceiver & 19 & 24 & 32 & 36 & 31 & Non & non \\
\hline 3D-DWT ENCRYPTION- transceiver & 13 & 15 & 25 & 31 & 19 & Non & non \\
\hline
\end{tabular}

\section{Conclusion}

The scrambling possibilities based on 3D-DWT matrix show the following features are:

a) Bandwidth is preserved.

b) There is no noise expansion, and quality of the recovered is preserved.

c) There exist fast algorithms, chapter four contains description to the forward algorithm.

d) Inverse transform is found easily, and has the same fast algorithm. Chapter four contains description to the reversed algorithm.

e) The encrypted data is meaningless and thus the residual intelligibility is considerably very low.

f) Permutation are better than inserted dummy components.

g) cryptanalytic efforts are considerably increased due to altered data components in such away will take infinite time to retrieve data if they do not know the structure of the system or one of its limits;

h) Implementation of the new scrambling concept into all existing data scramblers is straightforward, i.e., this concept is fully compatible with conventional systems.

\section{References}

[1] Amara Graps, "An Introduction to Wavelets," IEEE Computational Science and Engineering, vol. 2, num. 2, published by the IEEE Computer Society, Summer 1995.

[2] S. Mallat. A Theory for Multiresolution Signal Decomposition: the Wavelet Representation. IEEE Transaction on Pattern Analysis and Machine Intelligence, 11, pp. 674-693, 1989 H. Simpson, Dumb Robots, 3rd ed., Springfield: UOS Press, 2004, pp.6-9.

[3] M. Vishwanath and C. Chakrabarti, "A VLSI Architecture for Real-Time Hierarchical Encoding/Decoding of Video using the Wavelet Transform," IEEE International Conference on Acoustics, Speech and Signal Processing (ICASSP '94), Adelaide, Australia, vol. 2, April 19-22, 1994, pp. 401-404.

[4] M. Weeks, et al, "Discrete Wavelet Transform: Architectures, Design and Performance Issues," Journal of VLSI Signal Processing 35, 155-178, 2003.

[5] Md. ShoaiburRahmanl, Md. AynalHaque, "Introduction to a Novel Wavelet," IEEE/OSA/IAPR International Conference on Informatics, Electronics \& Vision, 2012. 
[6] V.Senk, V. D. Deli'c,V. S. Milo`sevi'c, "A New Speech Scrambling Concept Based on Hadamard Matrices," IEEE SIGNAL PROCESSING LETTERS, VOL. 4, NO. 6, JUNE 1997.

[7] H. Ojanen. "Orthonormal compactly supported wavelets with optimal sobolev regularity. Applied and Computational Harmonic Analysis, 10, pp. 93-98,2001.

[8] A.R. Calderbank, I. Daubechies, W. Sweldens, and B.L. Yeo. "Wavelet transforms that map integers to integers,". Appl. Comp. Harm. Anal., 5 (3), pp. 332-369,1998.

[9] Shannon, C. E., "Communication Theory of Secrecy Systems, "Bell System Technical Jo urnal, Vol. 28, 1949, pp. 656-715.

[10] Dr. Jameel Ahmed, "Transform-Domain and DSP Based Secure Speech Communication". Ph.D. dissertation, Hamdard Institute of Information Technology, 2007.

[11] D.J.H. Garling, D. Gorenstein, T. Tom Dieck, P. Walters, "WAVELETS AND OPERATORS,", Cambridge University Press 1992

[12] Tuan Van Pham, "Wavelet Analysis for Robust Speech Processing and Applications," VDM Verlag, Germany, 2008.

[13] StephaneMallat, "A Wavelet Tour of Signal Processing," Elsevier, 1999.

[14] Richard E. Blahut, "Fast Algorithms for Signal Processing," CAMBRIDGE UNIVERSITY PRESS, 2010.
[15] John J. Benedetto, "Applied and Numerical Harmonic Analysis: Frames and Bases," Birkha"user Boston, 2008.

[16] Mladen Victor W. ,"Adapted Wavelet Analysis from Theory to Software," A K Peters,Ltd 1994.

[17] Michel Misiti, Yves Misiti, Georges Oppenheim, Jean-Michel Poggi "Wavelet Toolbox For Use with MATLAB ${ }^{\circledR}$," The MathWorks, Inc, 2002.

[18] Alfred Mertins, "Signal Analysis: Wavelets, Filter Banks, Time-Frequency Transforms and Applications,",Mertins, Signaltheorie, 1996.

[19] Ali N. Akansu, Michael J. Medley, "WAVELET, SUBBAND AND BLOCK TRANSFORMS IN COMMUNICATIONS AND MULTIMEDIA," Kluwer Academic / Plenum Publishers, New York, 2002.

[20] A.Jensen, A.la Cour-Harbo, "Ripples in Mathematics The Discrete Wavelet Transform,", Springer.Verlag Berlin Heidelberg 2001.

[21] CHARLESK.CHUI, "An Introduction to Wavelets,", Academic Press 1992.

[22] C. Sidney Burrus, Ramesh A. Gopinath, and .HaitaoGuo, "Introduction to Wavelets and Wavelet Transforms," PrenticeHall, Inc., 1998.

[23] "Wavelets and Multiscale Analysis Theory and Applications,"SpringerScience+Business Media, LLC, 2011. 